\title{
Reversal of the Adverse Chronic Effects of the Unsaturated Derivative of Valproic Acid-2-n- Propyl-4-Pentenoic Acid-on Ketogenesis and Liver Coenzyme A Metabolism by a Single Injection of Pantothenate, Carnitine, and Acetylcysteine in Developing Mice
}

\author{
JEAN HOLOWACH THURSTON AND RICHARD E. HAUHART \\ Department of Pediatrics [J.H.T., R.E.H.] and Neurology and Neurological Surgery (Neurology) [J.H.T], \\ Washington University School of Medicine, St. Louis Children's Hospital, St. Louis, Missouri 63110
}

\begin{abstract}
Like treatment with the parent compound valproic acid (VPA), acute and/or chronic treatment with the unsaturated derivative, 2-n-propyl-4-pentenoic acid (4-en-VPA), decreased ketogenesis and lowered free CoA, acetyl $\mathrm{CoA}$, and free carnitine levels in the livers of normal developing mice. Concomitantly, there were manifold increases in the content of medium-chain acyl CoA esters (4-en-VPA CoA and 4-en-VPA CoA metabolites). Acute cotreatment of 4-en-VPA-treated animals with pantothenate, carnitine, and acetylcysteine caused significant amelioration of these metabolic aberrations. In animals chronically treated with 4-en-VPA, a single injection of pantothenate, carnitine, and acetylcysteine returned the 4-en-VPA-depressed levels of $\beta$-hydroxybutyrate in plasma and free $\mathrm{CoA}$ and acetyl $\mathrm{CoA}$ in liver to normal. These findings support the hypothesis that VPA- and 4-en-VPA-induced hepatic dysfunction is produced by $\mathrm{CoA}$ sequestration rather than by irreversible inhibition by alkylation of the enzymes of fatty acid $\beta$-oxidation by reactive intermediates. The findings also support the important but little-known role of carnitine in CoA metabolism-carnitine relieves the inhibition of pantothenate kinase, the ratecontrolling first enzyme in the pathway of $\mathrm{CoA}$ synthesis by its product, free CoA, and by CoA esters. (Pediatr Res 33: 72-76, 1993)
\end{abstract}

\section{Abbreviations}

VPA, valproic acid (2-n-propylpentanoic acid)

4-en-VPA, 2-n-propyl-4-pentenoic acid

4-PA, 4-pentenoic acid

Inhibition of fatty acid oxidation by VPA is an invariate finding both in vitro and in vivo (1-8 and many others). Two major mechanisms for this adverse action have been proposed. One relates to the depletion of free $\mathrm{CoA}$ in liver due to its sequestration in poorly metabolized valproyl $\mathrm{CoA}$ and valproyl CoA derivatives $(3,9)$. The other hypothesis is that highly reactive

Received June 19, 1992; accepted September 2, 1992.

Correspondence: Jean Holowach Thurston, B.A., M.D., Department of Pediatrics, Washington University School of Medicine, St. Louis Children's Hospital, One Children's Place, St. Louis, MO 63110.

Supported in part by the Abbott Laboratories, Chicago, IL, and the Allen P and Josephine B. Green Foundation, Mexico, MO. unsaturated intermediates of VPA alkylate, and thereby cause irreversible inhibition of, key enzymes of $\beta$-oxidation $(10,11)$.

Gerber et al. (12) were among the first to report VPA-associated irreversible hepatic failure in young infants and children. Because of the structural similarity of the unsaturated metabolite of VPA, 4-en-VPA, to two well-known hepatotoxins-4-pentenoic acid and toxic hypoglycin A metabolites $(13,14)$ - the authors believed that 4-en-VPA played a major role in the pathogenesis of VPA-induced hepatotoxicity. The present study is a test of this hypothesis in normal developing mice. Its results appear to be applicable to an explanation of the mechanism of action of these other hepatotoxins. Because we have found that coadministration of pantothenate and carnitine with VPA ameliorated its adverse effects on ketogenesis and liver CoA metabolism (15), effects of a similar regimen in acutely and chronically 4-en-VPAtreated mice were also examined.

\section{MATERIALS AND METHODS}

Pantothenate (D-pantothenic acid, hemicalcium salt), and $\mathrm{N}$ acetyl-L-cysteine were purchased from Sigma Chemical Co., St. Louis, MO. Solutions of N-acetyl-L-cysteine were neutralized to pH 7.0 with $\mathrm{NaOH}$ just before use. 4-en-VPA and sterile $0.9 \%$ $\mathrm{NaCl}$ were gifts of Abbott Laboratories, Chicago, IL; L-carnitine was a gift of Sigma-Tau, Rome, Italy.

Preparation of Animals. All animal experiments were performed with the highest standards of humane care.

Acute 4-en-VPA Administration and Cotreatment with Pantothenate, Carnitine, and Acetylcysteine. Five litters of 4- to 8-dold Swiss Webster mice (total 34) were used. The mean age $( \pm \mathrm{SEM})$ of these mice was $5.2 \pm 0.6 \mathrm{~d}$; their mean weight was $3.52 \pm 0.22 \mathrm{~g}$. Weight-matched littermates were injected s.c. with $20-30 \mathrm{mg} / \mathrm{kg}$ of $4-\mathrm{en}-\mathrm{VPA}$ in $20 \mathrm{~mL} / \mathrm{kg}$ of $0.9 \% \mathrm{NaCl}$ or an equivalent volume of $0.9 \% \mathrm{NaCl}$ (controls). Findings in experimental mice receiving 20 or $30 \mathrm{mg} / \mathrm{kg}$ of VPA or 4-en-VPA, acutely or chronically, were not significantly different; therefore, results were pooled. Other littermates received 4-en-VPA plus pantothenate (vitamin $\mathrm{B}_{5} ; \mathrm{CoA}$ precursor), $2 \mathrm{mmol} / \mathrm{kg}$; carnitine, $2.5 \mathrm{mmol} / \mathrm{kg}$; and acetylcysteine, $1 \mathrm{mmol} / \mathrm{kg}$. All animals were killed by decapitation $90 \mathrm{~min}$ after injection. Blood was collected from the severed neck vessels and the body was plunged into liquid nitrogen with rapid stirring to effect quick freezing. We have previously determined that liver metabolite levels in neonatal and developing mice of this age frozen in liquid nitrogen were not different from those found in neonatal and developing 
mice frozen in liquid Freon $12\left(\mathrm{CCl}_{2} \mathrm{~F}_{2}\right)$ at its melting point of $150^{\circ} \mathrm{C}$ (unpublished data).

Chronic 4-en-VPA Administration and Treatment with Pantothenate, Carnitine, and Acetylcysteine. Two litters of 5- to 7-dold mice were used for this study (total 19 animals). The mean age of these mice on the final day of the experiment was $10.0 \pm$ $0.4 \mathrm{~d}$; their mean weight was $5.38 \pm 0.27 \mathrm{~g}$. The mice received 4-en-VPA $(20-30 \mathrm{mg} / \mathrm{kg})$ or $0.9 \% \mathrm{NaCl}$ as described above, once daily for $5 \mathrm{~d}$. On the last day, some 4-en-VPA-injected littermates were treated with pantothenate, carnitine, and acetylcysteine in the dosage given above. The animals were killed 90 min later. Subsequent methods of procedure were as described above.

Preparation of Plasma and Liver. Blood was promptly centrifuged at $4^{\circ} \mathrm{C}$. Plasma was deproteinized with 10 to 20 volumes of $0.5 \mathrm{M}$ perchloric acid. It was not necessary to neutralize the acid extracts for assay of plasma metabolites; aliquots of the acid extract required for fluorometric measurements of $\beta$-hydroxybutyrate did not change the $\mathrm{pH}$ of the reagent buffer or affect complete recovery of the standard. Liver was dissected free of membranes and visible blood vessels with sharp chisels in a cryostat at $-35^{\circ} \mathrm{C}$. Perchlorate extracts were prepared in a cold room at $-20^{\circ} \mathrm{C}(16)$. Extracts were stored at $-80^{\circ} \mathrm{C}$ until the time of assay.

Analytical Methods. $\beta$-Hydroxybutyrate. The plasma $\beta$-hydroxybutyrate level was measured by a fluorometric adaptation of the method of Williamson et al. (17).

Free COA and COA esters. Free CoA, acetyl CoA, and acyl $\mathrm{CoA}$ esters were measured by the cycling procedures of Kato (18) and McDougal and Dargar (19). Medium-chain acyl CoA esters were hydrolyzed by heating a portion of the perchloric acid extract for $60 \mathrm{~min}$ at $60^{\circ} \mathrm{C}$ in the presence of $6 \mathrm{mM}$ DTT and $70 \mathrm{mM}$ 2-amino-2-methyl-1-propanol buffer at pH 9.2 (19). The concentration of medium-chain acyl CoA esters was calculated by subtracting the previously determined concentrations of free $\mathrm{CoA}$ and acetyl CoA. The perchloric acid-insoluble precipitate was used for the measurement of long-chain acyl CoA esters.

Free carnitine and carnitine esters. Free carnitine was assayed fluorometrically by measuring the CoA formed (20) from the reaction of carnitine with acetyl $\mathrm{CoA}$ in the presence of carnitine acetyltransferase (EC 2.3.1.7). Short- and medium-chain acyl carnitine esters were hydrolyzed by heating a portion of the neutralized perchloric acid extract for $1 \mathrm{~h}$ at $37^{\circ} \mathrm{C}$ in the presence of $150 \mathrm{mM} N$-2-hydroxyethylpiperazine- $N^{\prime}$-2-ethanesulfonic acid; the $\mathrm{pH}$ of the solution was adjusted to approximately 13 with $10 \mathrm{~N} \mathrm{NaOH}(21,22)$. After neutralization with $5 \mathrm{~N} \mathrm{HCl}$, the total acid-soluble carnitine content was measured as described above. The concentration of short- and medium-chain acyl carnitine esters was calculated by subtracting the concentration of free carnitine. The levels of long-chain acyl carnitine esters were similarly determined using the perchloric acid-insoluble precipitate.

Statistical Analysis. The statistical significance of the difference between the means of control and experimental values in the acute and chronic studies was determined by a one-way analysis of variance, followed by Tukey's Honestly Significant Difference test (23) to identify differences between specific pairs of means.

\section{RESULTS}

Clinical. There were no apparent clinical side effects of acute or chronic 4-en-VPA administration; the behavior of the experimental animals could not be distinguished from that of their control littermates. At the end of $5 \mathrm{~d}$ of 4-en-VPA injections, the weight of the animals was not different from that of control littermates (not shown). At the time of decapitation, the animals in the chronic study were twice the age of those used for the acute study (10 versus $5 \mathrm{~d}$ ) and weighed $53 \%$ more.

Effect of Acute 4-en-VPA Alone and 4-en-VPA Plus Pantothenate, Carnitine, and Acetylcysteine on Ketogenesis and Liver
CoA and Carnitine Metabolism. Acute administration of 4-enVPA lowered the plasma $\beta$-hydroxybutyrate concentration $51 \%$ (Table 1). It should be recalled that because of the high fat content of maternal milk, ketonemia is physiologic in suckling rodents.

In liver, 4-en-VPA caused a $56 \%$ drop in the level of free CoA and a $70 \%$ decrease in the acetyl CoA content. Concomitantly, there was a 7-fold increase of medium-chain acyl CoA esters [4en-VPA CoA and 4-en-VPA CoA metabolites (3) and other medium-chain acyl CoA esters]. Seventy percent of the increase in medium-chain acyl CoA could be accounted for by the combined drop in free and acetyl CoA. As we have previously found for VPA (9), 4-en-VPA did not change the content of long-chain acyl CoA esters. There was a nearly 4-fold increase in the acyl CoA:free CoA concentration ratio.

The drop in the free carnitine level was similar to that of free CoA: $52 \%$. There was no change in the level of short- and medium-chain acyl carnitine esters. The long-chain acyl carnitine ester fraction almost tripled. The total carnitine content was unchanged. There was a 2.5 -fold increase in the acyl carnitine:free carnitine concentration ratio.

Coadministration of pantothenate, carnitine, and acetylcysteine with acute 4 -en-VPA prevented the decrease in plasma $\beta$ hydroxybutyrate levels seen with 4-en-VPA alone and caused significant increases in the contents of free CoA and acetyl CoA: 88 and $169 \%$, respectively. These values were still about $17 \%$ less than in controls. Pantothenate, carnitine, and acetylcysteine cotreatment induced a further $15 \%$ increase in the content of medium-chain acyl CoA esters. The total CoA content increased $31 \%$. Concomitantly, there was a $33 \%$ drop in the 4-en-VPAelevated acyl CoA:free CoA concentration ratio; the $34 \%$ decrease in the corresponding acyl carnitine:free carnitine ratio was not statistically significant.

Effect of Chronic 4-en-VPA Alone and Treatment with Pantothenate, Carnitine, and Acetylcysteine on Ketogenesis and Liver CoA Metabolism. Because the hepatotoxic effects of 4-en-VPA might worsen with time or might not have responded as well to treatment with pantothenate, carnitine, and acetylcysteine as in the acute study, the effects of chronic 4-en-VPA administration were examined (Table 2). Chronic 4-en-VPA lowered the plasma $\beta$-hydroxybutyrate concentration $38 \%$. The liver free CoA content also fell $38 \%$; acetyl CoA levels decreased $52 \%$. The content of medium-chain acyl CoA esters increased 14-fold. Only one half of the increase could be accounted for by the drop in free CoA and acetyl CoA. The total CoA content increased $29 \%$. The acyl CoA:free $\mathrm{CoA}$ ratio increased 5-fold.

Qualitatively and quantitatively, the effects of chronic 4-enVPA on liver carnitine metabolism were similar to those seen in the acute study. The $54 \%$ increase in the content of long-chain acyl carnitine esters did not quite reach statistical significance.

A single coinjection of pantothenate, carnitine, and acetylcysteine on the 5th (last) d of 4-en-VPA injections returned the 4en-VPA-depressed level of plasma $\beta$-hydroxybutyrate to normal. Levels of free CoA and acetyl CoA in liver were now also not different from the control values. The elevated medium-chain acyl CoA ester fraction was not reduced.

Effects of pantothenate, carnitine, and acetylcysteine on carnitine metabolism were similar to those seen in the acute study except that now the $60 \%$ reduction of the acyl carnitine:free carnitine ratio induced by the vitamin, carnitine, and acetylcysteine mixture was statistically significant.

\section{DISCUSSION}

As was the case for VPA (15), acute coadministration of pantothenate and carnitine ameliorated the ill effects of 4-enVPA on ketogenesis and liver CoA metabolism in developing mice. Additionally, a single injection of pantothenate, carnitine, and acetylcysteine brought about a reversal of the adverse hepatotoxic effects of chronic 4-en-VPA: plasma $\beta$-hydroxybutyrate 
Table 1. Effect of pantothenate, carnitine, and $N$-acetylcysteine on plasma $\beta$-hydroxybutyrate levels and liver CoA and carnitine metabolism in suckling mice treated acutely with 4-en-valproate*

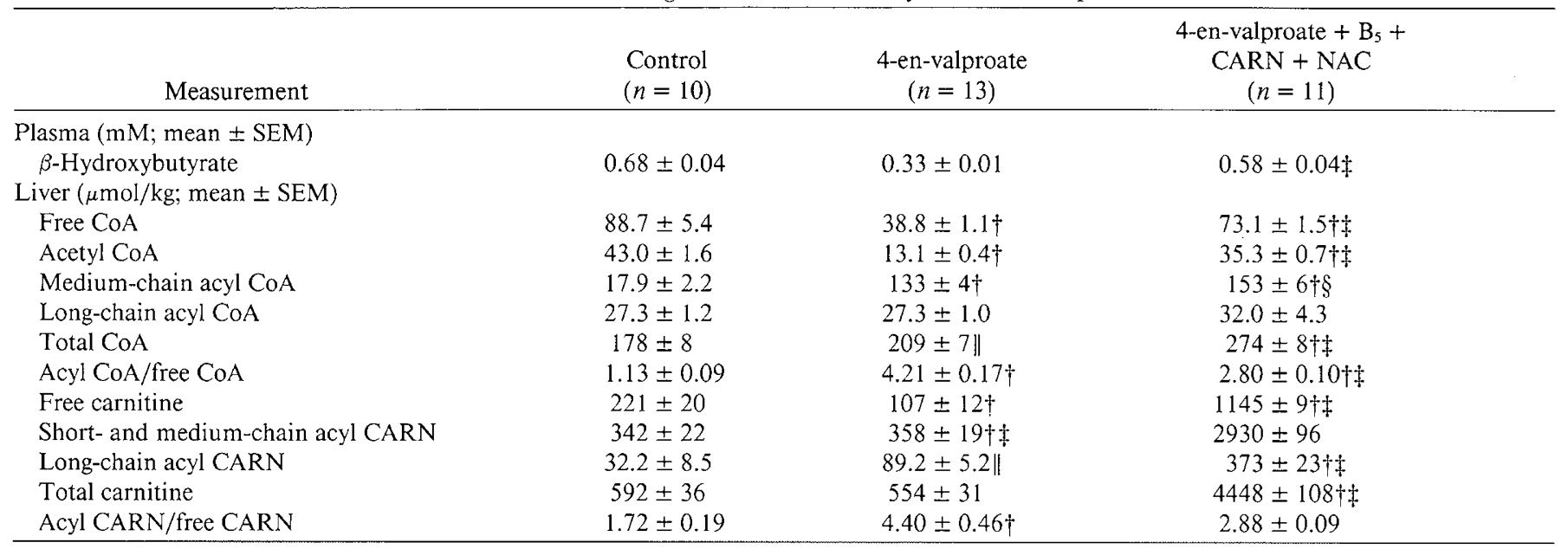

* Mice were treated as described in Materials and Methods. $\mathrm{B}_{5}$, pantothenic acid; CARN, carnitine; NAC, N-acetylcysteine.

$\dagger p$ vs control, $<0.01$.

$\$ p$ vs 4-en-valproate, $<0.01$.

$\S p$ vs 4-en-valproate, $<0.05$.

$\| p$ vs control, $<0.05$.

Table 2. Effect of pantothenate, carnitine, and $N$-acetylcysteine on plasma $\beta$-hydroxybutyrate levels and liver CoA and carnitine metabolism in suckling mice treated chronically with 4-en-valproate*

\begin{tabular}{|c|c|c|c|}
\hline Measurement & $\begin{array}{c}\text { Control } \\
(n=8) \\
\end{array}$ & $\begin{array}{c}\text { 4-en-valproate } \\
(n=7)\end{array}$ & $\begin{array}{c}\text { 4-en-valproate }+\mathrm{B}_{5}+ \\
\mathrm{CARN}+\mathrm{NAC} \\
(n=4)\end{array}$ \\
\hline \multicolumn{4}{|l|}{ Plasma (mM; mean \pm SEM) } \\
\hline \multicolumn{4}{|l|}{ Liver $(\mu \mathrm{mol} / \mathrm{kg} ;$ mean $\pm \mathrm{SEM})$} \\
\hline Free $\mathrm{CoA}$ & $131 \pm 8$ & $80.5 \pm 7.9 \dagger$ & $112 \pm 5 \S$ \\
\hline Acetyl CoA & $33.0 \pm 1.9$ & $15.9 \pm 1.3 \dagger$ & $38.6 \pm 3.6 \ddagger$ \\
\hline Total CoA & $190 \pm 9$ & $246 \pm 3 \dagger$ & $317 \pm 11+t$ \\
\hline Acyl $\mathrm{CoA} /$ free $\mathrm{CoA}$ & $\begin{array}{l}0.552 \pm \\
0.059\end{array}$ & $2.61 \pm 0.08 \dagger$ & $1.85 \pm 0.10 \dagger$ \\
\hline Free carnitine & $242 \pm 15$ & $121 \pm 17 \dagger$ & $1003 \pm 23+t$ \\
\hline Short- and medium-chain acyl CARN & $350 \pm 40$ & $462 \pm 14$ & $1833 \pm 224 \dagger t$ \\
\hline Long-chain acyl CARN & $71.3 \pm 6.7$ & $110 \pm 10$ & $350 \pm 23+\ddagger$ \\
\hline
\end{tabular}

* Mice were treated as described in Materials and Methods. Abbreviations are those used in Table 1.

$\dagger p$ ys control, <0.01.

$\ddagger p$ vs 4-en-valproate, $<0.01$.

$\S p$ vs 4-en-valproate, $<0.05$.

levels were returned to normal as were the depressed levels of free $\mathrm{CoA}$ and acetyl $\mathrm{CoA}$ in liver; only the medium-chain acyl CoA ester fraction remained elevated.

Differences in the levels of some of the metabolites in the control animals used for the acute and chronic studies are to be expected among the seven different litters of mice used for this research. Whether the older age and the greater weight at the time of decapitation of the mice used for the chronic study contributed to the observed differences cannot be said.

Kesterson et al. (7) and Granneman et al. (8) have compared the in vivo effects of chronic VPA and 4-en-VPA administration on liver intermediary metabolism in young rats. But neither the drug dosages, ages, nor species were comparable to the protocol used in the present study.

Supporting a role of 4-en-VPA in VPA-hepatotoxicity is the fact that chronic coadministration of VPA with phenobarbital in developing mice caused a significant worsening of the adverse effects seen in littermates treated with VPA alone (24). Pheno- barbital induces the formation of 4-en-VPA from VPA via the microsomal cytochrome P-450 system (25-27). Chronic cotreatment of young rats with VPA and phenobarbital doubled the plasma 4-en-VPA level and induced hepatic steatosis $(7,8)$. However, liver acetyl CoA levels were not reduced by 4-en-VPA, and inhibition of $\beta$-oxidation was considerably less, not more, severe in animals cotreated with phenobarbital and VPA than in animals receiving only VPA (7). The findings suggest that the steatogenic effect of phenobarbital in the livers of VPA-cotreated rats relates to some mechanism other than inhibition of $\beta$ oxidation (8). Metabolite changes in the livers of young 4-enVPA-injected mice suggested a lesser degree of inhibition of $\beta$ oxidation than with VPA (28).

The metabolic aberrations induced by chronic 4-en-VPA therapy were not more severe than those seen in the acutely treated animals. The finding may reflect a compensatory or adaptive response. In contrast with acute in vitro VPA studies, chronic VPA administration in developing and adult mice and rats 
induced significant in vivo elevations of the hepatic activities of enzymes of $\beta$-oxidation, ketogenesis, and amino acid metabolism (29-31). It is interesting that VPA consistently lowered hepatic levels of the substrates and/or cofactors related to these enzymic reactions $(3,5,7,9,32)$.

As mentioned earlier, based on the structural similarity of 4 en-VPA to 4-PA it was speculated that the hepatotoxicity of VPA might be linked to the formation of 4-en-VPA and further biotransformation to yield highly reactive electrophilic intermediates $(10,11,33)$. In rats injected with radioactive VPA or 4en-VPA and in hepatocytes, radioactivity was associated irreversibly with unidentified liver proteins (10).

4-PA and unsaturated metabolites inhibited 3-keto-acyl CoA thiolase (EC 2.3.1.16) and/or acetoacetyl CoA thiolase (EC 2.3.1.9) in rat heart mitochondria (34-36). However, Osmundsen et al. (37) could not demonstrate inhibition of acetoacetyl CoA thiolase (EC 2.3.1.9) by 4-PA in rat liver mitochondria. To our knowledge, there are no published studies of in vivo inhibition of liver 3-keto-acyl CoA thiolase or acetoacetyl CoA thiolase by VPA, 4-en-VPA, or other reactive species.

The observed beneficial in vivo effects of pantothenate, carnitine, and acetylcysteine in acute or chronic 4-en-VPA-induced hepatotoxicity should perhaps not be surprising. Inhibition of hepatic fatty acid $\beta$-oxidation by 4-PA in vitro was reversed by the addition of free CoA to the reaction mixture, with or without carnitine $(14,38,39)$. Like VPA and 4-en-VPA, 4-PA also lowered levels of free CoA and free carnitine in liver $(13,40)$, suggesting that its deleterious effect on liver metabolism was due, at least in part, to sequestration of free $\mathrm{CoA}$ and carnitine in relatively inert 4-pentenoyl $\mathrm{CoA}$ and carnitine esters.

We found equally beneficial effects in VPA-treated mice by cotreatment with pantothenic acid and carnitine with or without cysteine (15). Farrell et al. (41) have reported a striking effect of oral acetylcysteine in three children with VPA-induced hepatotoxicity. The beneficial effect was attributed to the action of acetylcysteine to conjugate VPA and reactive metabolites (42). Hepatic hydrolysis of acetylcysteine to liberate cysteine for CoA synthesis could also have contributed to the improvement of these children. Coinjection of acetylcysteine with VPA in our developing mice had no effect on the VPA-induced inhibition of ketogenesis (unpublished data).

The beneficial effect of carnitine in the treatment of organic acidemias is believed to be due to conversion of accumulated CoA esters of these acids via the action of carnitine acyltransferases to their corresponding carnitine derivatives. However, unlike the physiologic organic acids, VPA does not readily react with carnitine acyltransferase in rat liver (3). In VPA-treated mice, coadministration of a 28 -fold greater dose of carnitine had no effect on the depressed levels of ketone bodies in the blood and free CoA or acetyl CoA in liver (15). Nor did carnitine cotreatment, with or without pantothenate, lower the greatly elevated medium-chain acyl CoA ester fraction (including valproyl CoA and valproyl CoA metabolites) (15).

Carnitine esters of VPA or VPA metabolites may not readily be formed by human liver either. In children receiving valproate and carnitine, valproyl carnitine accounted for $<10 \%$ of the total acyl carnitines in urine (43). In another human study, valproyl carnitine esters in urine increased from $0.4 \%$ before carnitine supplementation to only $1 \%$ after (44).

It is also relevant that valproyl CoA is poorly hydrolyzed by medium-chain acyl CoA hydrolase activity in liver preparations from both control and valproate-fed rats and rabbits (45).

Pantothenate alone increased the levels of free $\mathrm{CoA}$ and acetyl CoA in liver after VPA-induced depression but had no effect on the reduced concentration of $\beta$-hydroxybutyrate in plasma (ketogenesis) (15). Only after the addition of carnitine with pantothenate did we see stimulation of ketogenesis and further statistically significant increases of free $\mathrm{COA}$ and acetyl $\mathrm{CoA}$ in liver. This cooperative action may relate to the important but less wellknown role of carnitine in CoA metabolism. In heart, liver, and kidney, pantothenic acid kinase (EC 2.7.1.33), is strongly inhibited by the end product, free CoA and its acyl esters (46-48). Free carnitine deinhibits the CoA-inhibited enzyme $(48,49)$. The need for the addition of carnitine with pantothenate to increase ketogenesis and to elevate the depressed levels of free $\mathrm{COA}$ and acetyl CoA in the livers of our VPA and 4-en-VPA-treated mice strengthens these observations.

The amelioration or curative effects of coadministration of pantothenate and carnitine seen with VPA- or 4-en-VPA support the CoA depletion theory of valproate hepatotoxicity. Although direct inhibition (by alkylation) of enzymes of $\beta$-oxidation and other important enzymes may play a role in the production of VPA-induced hepatotoxicity, no evidence requiring such an explanation was seen in this study. Specifically, treatment with pantothenate, carnitine, and acetylcysteine produced increased levels of acetyl CoA and free $\mathrm{CoA}$ in liver and $\beta$-hydroxybutyrate in plasma, but it did not reduce the level of the medium-chain acyl CoA ester fraction where toxic 4-en-VPA and derivatives would be expected to be.

Acknowledgments. The authors thank Dr. David B. McDougal, $\mathrm{Jr}$. for his continued interest and encouragement and his critical review of the manuscript.

\section{REFERENCES}

1. Matsumoto I, Kuhara T, Yoshino M 1976 Metabolism of branched medium chain length fatty acid. II. $\beta$-oxidation of sodium dipropylacetate in rats. Biomed Environ Mass Spectrom 3:235-240

2. Mortensen PB 1980 Inhibition of fatty acid oxidation by valproate. Lancet 2:856-857

3. Becker C-M, Harris RA 1983 Influence of valproic acid on hepatic carbohydrate and lipid metabolism. Arch Biochem Biophys 223:381-392

4. Turnbull DM, Bone AJ, Bartlett K, Koundakjian PP, Sherratt HSA 1983 The effects of valproate on intermediary metabolism in isolated rat hepatocytes and intact rats. Biochem Pharmacol 32:1887-1892

5. Coudé FX, Grimber G, Pelet A, Benoit Y 1983 Action of the antiepileptic drug, valproic acid, on fatty acid oxidation in isolated hepatocytes. Biochem Biophys Res Commun 115:730-736

6. Thurston JH, Carroll JE, Norris BJ, Hauhart RE, Schiro JA 1983 Acute in vivo and in vitro inhibition of palmitic acid and pyruvate oxidation by valproate and valproyl-CoA in livers of infant mice. Ann Neurol 14:384385 (abstr)

7. Kesterson JW, Granneman GR, Machinist JM 1984 The hepatotoxicity of valproic acid and its metabolites in rats. I. Toxicologic, biochemical and histopathologic studies. Hepatology 4:1143-1152

8. Granneman GR, Wang S-I, Kesterson JW, Machinist JM 1984 The hepatotoxicity of valproic acid and its metabolites in rats. II. Intermediary and valproic acid metabolism. Hepatology 4:1153-1158

9. Thurston JH, Carroll JE, Hauhart RE, Schiro JA 1985 A single therapeutic dose of valproate affects liver carbohydrate, fat, adenylate, amino acid, coenzyme $\mathrm{A}$, and carnitine metabolism in infant mice: possible clinical significance. Life Sci 36:1643-1651

10. Porubek DJ, Grillo MP, Baillie TA 1989 The covalent binding to protein of valproic acid and its hepatotoxic metabolite, 2-n-propyl-4-pentenoic acid, in rats and in isolated rat hepatocytes. Drug Metab Dispos 17:123-130

11. Rettenmeier AW, Gordon WP, Prickett KS, Levy RH, Baillie TA 1986 Biotransformation and pharmacokinetics in the rhesus monkey of 2-npropyl-4-pentenoic acid, a toxic metabolite of valproir acid. Drug Metab Dispos 14:454-464

12. Gerber N, Dickinson RG, Harland RC, Lynn RK, Houghton D, Antonias JI, Schimschock JC 1979 Reye-like syndrome associated with valproic acid therapy. J Pediatr 95:142-144

13. Holland PC, Sherratt HSA 1973 Biochemical effects of the hypoglycaemic compound pent-4-enoic acid and related non-hypoglycaemic fatty acids. Effects of the free acids and their carnitine esters on coenzyme A-dependent oxidations in rat liver mitochondria. Biochem J 136:157-171

14. Bressler R, Corredor C, Brendel K 1969 Hypoglycin and hypoglycin-like compounds. Pharmacol Rev 21:105-130

15. Thurston JH, Hauhart RE 1992 Amelioration of adverse effects of valproic acid on ketogenesis and liver coenzyme A metabolism by cotreatment with pantothenate and carnitine in developing mice: possible clinical significance. Pediatr Res 31:419-423

16. Lowry OH, Passonneau JV, Hasselberger FX, Schulz DW 1964 Effect of ischemia on known substrates and cofactors of the glycolytic pathway in brain. J Biol Chem 239:18-30

17. Williamson DH, Mellanby J, Krebs HA 1962 Enzymatic determination of $\mathrm{D}(-)-\beta$-hydroxybutyric acid and acetoacetic acid in blood. Biochem $\mathrm{J} 82: 90-$ 98

18. Kato T 1975 CoA cycling: an enzymatic amplification method for determination of CoASH and acetyl CoA. Anal Biochem 66:372-392 
19. McDougal Jr DB, Dargar RV 1979 A spectrophotometric cycling assay for reduced coenzyme $A$ and its esters in small amounts of tissue. Anal Biochem 97:103-115

20. Tubbs PK, Garland PB 1969 Assay of coenzyme A and some acyl derivatives. In: Lowenstein JM (ed) Methods in Enzymology, Vol 13. Academic Press, New York, pp 535-551

21. McGarry JD, Foster DW 1976 An improved and simplified radioisotopic assay for the determination of free and esterified carnitine. J Lipid Res 17:277281

22. Christiansen RZ, Bremer J 1976 Active transport of butyrobetaine and carnitine into isolated liver cells. Biochim Biophys Acta 448:562-577

23. Tukey JW 1953 The problem of multiple comparisons. Ditto, Princeton University, Princeton, NJ. Cited by RE Kirk 1968 Experimental design: Procedures for the Behavioral Sciences. Brooks/Cole, Belmont, CA, pp 6998

24. Thurston JH, Hauhart RE 1991 Polytherapy aggravates valproate hepatotoxicity in normal suckling mice. Ann Neurol 30:448(abstr)

25. Porubek DJ, Barnes H, Meier GP, Theodore LJ, Baillie TA 1989 Enantiotopic differentiation during the biotransformation of valproic acid to the hepatotoxic olefin 2-n-propyl-4-pentenoic acid. Chem Res Toxicol 2:35-40

26. Rettie AE, Rettenmeier AW, Howald WN, Baillie TA 1987 Cytochrome P. 450 -catalyzed formation of $\Delta^{4}$-VPA, a toxic metabolite of valproic acid. Science 235:890-893

27. Rettie AE, Boberg M, Rettenmeier AW, Baillie TA 1988 Cytochrome P-450catalyzed desaturation of valproic acid in vitro. Species differences, induction effects, and mechanistic studies. J Biol Chem 263:13 733-13 738

28. Thurston $\mathrm{JH}$, Hauhart RE 1991 Effects of chronic valproate and its unsaturated 4-en derivative on selected aspects of $\beta$-oxidation in infant mouse liver. Pediatr Res 29:199A(abstr)

29. Thurston JH, Hauhart RE 1986 Hepatic toxicity of valproic acid: do inborn errors of liver metabolism underlie hepatic fatalities in some children? Ann Neurol 20:400(abstr)

30. Horie S, Suga $T 1985$ Enhancement of peroxisomal $\beta$-oxidation in the liver of rats and mice treated with valproic acid. Biochem Pharmacol 34:1357-1362

31. Cotariu D, Evans S, Zaidman JL 1985 Effects of sodium valproate on subcellular fraction enzymes in rat liver. Enzyme 34:196-200

32. Coudé FX, Grimber G, Parvy P, Rabier D, Petit F 1983 Inhibition of ureagenesis by valproate in rat hepatocytes: role of $\mathrm{N}$-acetylglutamate and acetyl CoA. Biochem J 216:233-236

33. Rettenmeier AW, Prickett KS, Gordon WP, Bjorge SM, Chang S-L, Levy RH, Baillie TA 1985 Studies on the biotransformation in the perfused rat liver of 2-n-propyl-4-pentenoic acid, a metabolite of the antiepileptic drug valproic acid. Drug Metab Dispos 13:81-96

34. Holland PC, Senior AE, Sherratt HSA 1973 Biochemical effects of the hypo- glycaemic compound pent-4-enoic acid and related non-hypoglycaemic fatty acids. Biochem J 136:173-184

35. Fong JC, Schulz $\mathrm{H} 1978$ On the rate-determining step of fatty acid oxidation in heart. Inhibition of fatty acid oxidation by 4-pentenoic acid. J Biol Chem 253:6917-6922

36. Schulz H 1983 Metabolism of 4-pentenoic acid and inhibition of thiolase by metabolites of 4-pentenoic acid. Biochemistry 22:1827-1832

37. Osmundsen H, Billington D, Sherratt HSA 1975 The effects of pent-4-enoate and methylenecyclopropylacetate on some enzymes of $\beta$-oxidation in rat liver mitochondria in vivo and in vitro. Biochem Soc Trans 3:331-333

38. Brendel K, Corredor CF, Bressler R 1969 The effect of 4-pentenoic acid on fatty acid oxidation. Biochem Biophys Res Commun 34:340-347

39. Fukami MH, Williamson JR 1971 On the mechanism of inhibition of fatty acid oxidation by 4-pentenoic acid in rat liver mitochondria. J Biol Chem 246:1206-1212

40. Corredor C, Brendel K, Bressler R 1967 Studies on the mechanism of the hypoglycemic action of 4-pentenoic acid. Proc Natl Acad Sci USA 58:22992306

41. Farrell K, Abbott FS, Junker AK, Waddell JS, Pippenger CE 1989 Successful treatment of valproate hepatotoxicity with $\mathrm{N}$-acetylcysteine. Epilepsia 30:700(abstr)

42. Kassahun K, Farrell K, Abbott F 1991 Identification and characterization of the glutathione and $\mathrm{N}$-acetylcysteine conjugates of (E)-2-propyl-2,4-pentadienoic acid, a toxic metabolite of valproic acid, in rats and humans. Drug Metab Dispos 19:525-535

43. Millington DS, Bohan TP, Roe CR, Yergey AL, Liberato DJ 1985 Valproylcarnitine: a novel drug metabolite identified by fast atom bombardment and thermospray liquid chromatography-mass spectrometry. Clin Chim Acta 145:69-76

44. Melegh B, Kerner J, Jaszai V, Bieber LL 1990 Differential excretion of xenobiotic acyl-esters of carnitine due to administration of pivampicillin and valproate. Biochem Med Metab Biol 43:30-38

45. Moore KH, Decker BP, Schreefel FP 1988 Hepatic hydrolysis of octanoyl-CoA and valproyl-CoA in control and valproate-fed animals. Int $J$ Biochem 20:175-178

46. Karasawa T, Yoshida K, Furukawa K, Hosoki K 1972 Feedback inhibition of pantothenate kinase by coenzyme $A$ and possible role of the enzyme for the regulation of cellular coenzyme A level. J Biochem 71:1065-1067

47. Halvorsen O, Skrede S 1982 Regulation of the biosynthesis of $\mathrm{COA}$ at the level of pantothenate kinase. Eur J Biochem 124:211-215

48. Fisher MN, Robishaw JD, Neely JR 1985 The properties and regulation of pantothenate kinase from rat heart. J Biol Chem 260:15745-15751

49. Fisher MN, Neely JR 1985 Regulation of pantothenate kinase from various tissues of the rat. FEBS Lett 190:293-296 